\title{
A Two-Layered Suspension Blood Flow Through a Composite Stenosis
}

\author{
Alana R. Sankar ${ }^{1}$, Sreedhara Rao Gunakala ${ }^{1}$ \& Donna M. G. Comissiong ${ }^{1}$ \\ ${ }^{1}$ Department of Mathematics and Statistics, The University of the West Indies, Trinidad and Tobago \\ Correspondence: Alana R. Sankar, Department of Mathematics and Statistics, The University of the West Indies, \\ Trinidad and Tobago. Tel: 1-868-662-2002 ext. 83553. E-mail: alana.sankar@sta.uwi.edu
}

Received: August 4, 2013 Accepted: October 3, 2013 Online Published: October 25, 2013

doi:10.5539/jmr.v5n4p26 URL: http://dx.doi.org/10.5539/jmr.v5n4p26

\begin{abstract}
Blood flow in the presence of a composite stenosis is investigated by examining the effects that red cell concentration, stenosis height and a peripheral layer have on blood flow characteristics. A two-layered model with a particle-fluid suspension in the core region and a peripheral plasma layer without any particles is used to represent blood. Expressions for three flow characteristics (impedance, wall shear stress and shear stress at the stenosis throat) are derived. Flow impedance increases with increasing hematocrit, stenosis height and diameter of the vessel but decreases with increasing tube length. The shear stress on the wall increases with increasing hematocrit, stenosis height and diameter of the vessel. Trends in the shear stress at the stenosis throat and impedance are similar with the variation of any parameter. The two-fluid model's flow characteristics are lower than those of the one-fluid model. In the analysis of the particle-fluid suspension the flow characteristics there were higher than when flow is considered to be particle-free. This knowledge of how the peripheral layer affects blood flow characteristics can aid in the understanding of diseased arterial systems.
\end{abstract}

Keywords: composite stenosis, blood, impedance, shear stress, hematocrit

\section{Introduction}

Studies of blood flow through stenotic arteries help scientists to understand cardiovascular diseases and allow for improved diagnostics of these diseases. Cardiovascular diseases arise out of a disorder of the cardiovascular system. The cardiovascular system, also known as the circulatory system, encompasses the heart and blood vessels of the body. It transports oxygenated blood from the left side of the heart via arteries of decreasing size down to the capillaries (the narrowest arteries). Here, there is an exchange of oxygen (and other nutrients) for carbon dioxide (and other waste products) which are then transported back to the heart through progressively enlarging veins to be re-oxygenated in the alveoli of the lungs. According to the World Health Organisation [WHO] (2013), an estimated 17 million people die annually of cardiovascular related complications, thus making it a popular subject of scientific research (World Health Organisation [WHO], 2013). It is predicted that cardiovascular diseases will remain the single leading cause of death worldwide with 23.3 million deaths by 2030 (WHO, 2013). The majority of deaths are caused by coronary heart disease which is disease of the blood vessels supplying the heart muscle or cerebrovascular disease which is disease of the blood vessels supplying the brain. Blood flow to the heart or the brain becomes blocked and usually leads to the occurence of a heart attack or a stroke respectively (WHO, 2013). One of the major factors that lead to the prevalence of these two groups of cardiovascular diseases is the presence of stenosis in blood vessels.

When an abnormal growth appears under diseased conditions in any location of the cardiovascular system such that it narrows any body, tube, orfice or passage, it is referred to by the medical term, stenosis (Young, 1979). Many researchers studied the effect that stenosis has on blood flow characteristics after the early investigations of Mann, Hennrick, Essex, and Blades (1938). These include the important contributions of Young (1968), Lee and Fung (1970), Nerem (1974), MacDonald (1979), Shukla and Coworkers (1980), Srivastava (1985), Haldar (1985), Chaturani and Samy (1986), Bitoun and Bellet (1986), Chakravarty (1987), Srivastava and Saxena (1994). Some studies conducted have treated stenosis as a single stenosis being either symmetric, bell shaped or in shape, while others have considered cases non-symmetric where stenosis develops in series (multiple stenosis) or overlapping, irregularly shaped or even composite in nature. 
An average adult has about five litres of blood which accounts for 7\% of the human body (Guyton \& Hall, 2006). Since blood is a suspension of corpuscles, it does not always exhibit the characteristics of a Newtonian fluid especially in tubes with small diameters (Charm \& Kurland, 1974; Hersey, Byrnes, \& Roam, 1964; Haynes, 1960). Based on Haynes' (1960) theoretical analysis and Cokelet's (1972) experimental observations, a singlephase homogeneous viscous fluid analysis cannot be used to describe blood flow in blood vessels of diameter $\leq$ $1000 \mu \mathrm{m}$ (Haynes, 1960; Cokelet, 1972). Generally in human blood, the hematocrit (red cell volume fraction) is approximately $45 \%$ and therefore has a strong influence on the flow properties of blood. The discovery that red blood cells (erythrocytes) should be treated as discrete particles was made in 1972 by Skalak (1972). L. M. Srivastava and V. P. Srivastava (1983) proposed a two-phase macroscopic model (i.e. a suspension of red cells in plasma) for blood in small vessels (of diameter $\leq 2400 \mu \mathrm{m}$ ) where the individuality of the red cells (of diameter $8 \mu \mathrm{m}$ ) is significant (L. Srivastava 1983; V. Srivastava, 1983). Srivastava (2007) discussed briefly suspension modeling of blood flow (Srivastava, 2007). Also, Bugliarello and Sevilla (1970), Cokelet (1972) and Thurston (1989) provided experimental evidence of the existence of a layer of plasma containing no cells located layer near the walls and a core region containing all the erythrocytes suspended in plasma (Bugliarello \& Sevilla, 1970; Cokelet, 1972; Thurston, 1989). Thus a realistic description of blood flow through small vessels should involve this two-layered model.

The significance of the peripheral layer increases as the flow in blood vessels of decreasing size is considered (Srivastava, 2007). Srivastava and Saxena (1997) performed an analysis of the effect of mild stenosis on blood flow using this model. Work done also considered the case of an overlapping constriction (Srivastava, Rastogi, \& Vishnoi, 2010). A two-layered model of blood flow through a composite stenosis was considered by Joshi et al. (2009) but this model consisted of two Newtonian fluids of different viscosities in which the effect of the hematocrit was ignored (P. Joshi, Pathak, \& B. Joshi, 2009). The inclusion of the hematocrit becomes important when analysing flow in small blood vessels. Blood flow through a composite stenosis was studied by Medhavi et al. (2012) for a two-phase macroscopic flow but the existence of a cell-free plasma (Newtonian viscous fluid) layer near the walls was not taken into consideration (Medhavi, R. Srivastava, Ahmad, \& V. Srivastava, 2012).

Therefore, in this paper, the flow of blood through small vessels in the presence of a composite stenosis will be examined using a two-layered model with a peripheral layer and a core region of erythrocytes in plasma, which is an improvement on the exisiting models. The study of a composite stenosis is important since the shape of the stenosis which is manifested varies. The two-layered model for blood flow provides a more realistic model for flow in small arteries since the existance of the peripheral layer and the red blood cells in the plasma can no longer be neglected. This model allows the investigation of the effects of pheripheral layer, stenosis height and hematocrit on the blood flow characteristics with a composite stenosis being present. The negative effect that this stenosis has on the vessels of the cardiovascular system is seen.

\section{Formulation of the Problem}

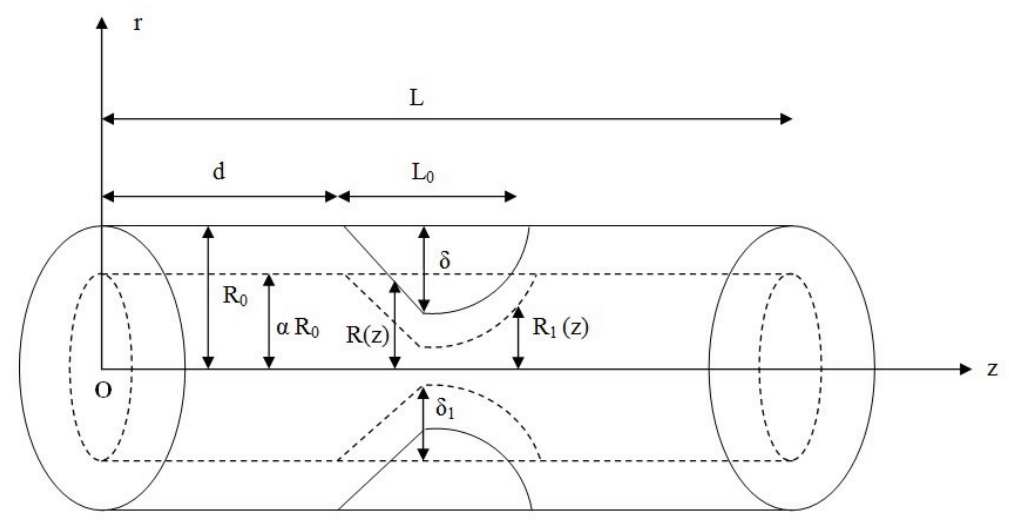

Figure 1. The geometry of the composite stenosis

Consider an axisymmetrical flow through an artery. The artery is assumed to be uniform, rigid and circular. The composite stenosis is mild, axially nonsymmetric and radially symmetric. The wall near to the stenosis development is assumed to be solid. Entrance, end and special wall effects are not considered due to the assumption 
that the length of the artery is much greater than its radius. A two-layered model is used to represent blood. It consists of a central layer of radius $R_{1}$ of erythrocytes suspended in plasma and a Newtonian, viscous fluid layer which is the peripheral plasma layer of thickness $\left(R-R_{1}\right)$. The geometry of the stenosis manifested, as shown in Figure 1, can be described as (Joshi et al., 2009)

$$
\begin{aligned}
\frac{\left(R(z), R_{1}(z)\right)}{R_{0}} & =(1, \alpha)-\frac{2}{R_{0} L_{0}}\left(\delta, \delta_{1}\right)(z-d) ; d \leq z \leq d+\frac{L_{0}}{2} \\
& =(1, \alpha)-\frac{\left(\delta, \delta_{1}\right)}{2 R_{0}}\left\{1+\cos \frac{2 \pi}{L_{0}}\left(z-d-\frac{L_{0}}{2}\right)\right\} ; d+\frac{L_{0}}{2} \leq z \leq d+L_{0} \\
& =(1, \alpha) ; \text { otherwise. }
\end{aligned}
$$

where $R \cong R(z)$ is the radius of the tube with constriction and $R_{0}$ are the radius of the tube without any constriction. The length of the tube is denoted by $L$ and the length of the stenosis by $L_{0}$. The stenosis starts at $z=d$. In the unobstructed region, the ratio of the radius of the central core to that of the tube is $\alpha$. The maximum height of the stenosis and bulging of the interface at the location where $z=d+\frac{L_{0}}{2}$ in the stenotic region is $\left(\delta, \delta_{1}\right)$.

In the core region, $\left(0 \leq r \leq R_{1}\right)$, a two-phase macroscopic model is used. Since blood has a very complicated structure additional assumptions must be made to simplify the analysis (L. Srivastava 1983; V. Srivastava, 1983):

(i) the red cell is spherical in shape and rigid,

(ii) the artery wall is rigid and of infinite length,

(iii) the flow has rotational symmetry,

(iv) a modified Stoke's drag law governs the interaction between the two phases,

(v) hematocrit is constant,

(vi) red cell-cell interaction and brownian motion is neglected.

The governing equations (both linear momentum and conservation of mass) for both the fluid and particle phases (using a continuum approach) are expressed as (L. Srivastava 1983; V. Srivastava, 1983; Drew, 1979)

$$
\begin{gathered}
\text { Fluid phase }\left\{\begin{array}{l}
(1-C) \rho_{f}\left(\frac{\partial u_{f}}{\partial t}+u_{f} \frac{\partial u_{f}}{\partial z}+v_{f} \frac{\partial u_{f}}{\partial r}\right)=-(1-C) \frac{\partial p}{\partial z}+(1-C) \mu_{s}(C) \nabla^{2} u_{f}+C S\left(u_{p}-u_{f}\right), \\
(1-C) \rho_{f}\left(\frac{\partial v_{f}}{\partial t}+u_{f} \frac{\partial v_{f}}{\partial z}+v_{f} \frac{\partial v_{f}}{\partial r}\right)=-(1-C) \frac{\partial p}{\partial r}+(1-C) \mu_{s}(C)\left(\nabla^{2}-\frac{1}{r^{2}}\right) v_{f}+C S\left(v_{p}-v_{f}\right),(5) \\
\frac{1}{r} \frac{\partial}{\partial r}\left[r(1-C) v_{f}\right]+\frac{\partial}{\partial z}\left[(1-C) u_{f}\right]=0,
\end{array}\right. \\
\text { Particulate phase }\left\{\begin{array}{c}
C \rho_{p}\left(\frac{\partial u_{p}}{\partial t}+u_{p} \frac{\partial u_{p}}{\partial z}+v_{p} \frac{\partial u_{p}}{\partial r}\right)=-C \frac{\partial p}{\partial z}+C S\left(u_{f}-u_{p}\right), \\
C \rho_{p}\left(\frac{\partial v_{p}}{\partial t}+u_{p} \frac{\partial v_{p}}{\partial z}+v_{p} \frac{\partial v_{p}}{\partial r}\right)=-C \frac{\partial p}{\partial r}+C S\left(v_{f}-v_{p}\right), \\
\frac{1}{r} \frac{\partial}{\partial r}\left(r C v_{p}\right)+\frac{\partial}{\partial z}\left(C u_{p}\right)=0 .
\end{array}\right.
\end{gathered}
$$

Here $(r, z)$ are two-dimensional cylindrical polar coordinate with $z$ measured along the axis of the tube and $r$ measured normal to the tube axis, $\nabla^{2}=\frac{1}{r} \frac{\partial}{\partial r}\left(r \frac{\partial}{\partial r}\right)+\frac{\partial^{2}}{\partial z^{2}}$ is a two-dimensional Laplacian operator, $\left(u_{f}, v_{f}\right)$ and $\left(u_{p}, v_{p}\right)$ are the (axial, radial) components of the fluid and particle velocities. $C$ denotes the volume fraction density of the particles, $p$ is the pressure, $\mu_{s}(C) \simeq \mu_{s}$ is the mixture viscosity (apparent or effective viscosity), $S$ is the drag coefficient of interaction for the force exerted by one phase on the other, $\rho_{f}$ and $\rho_{p}$ are the actual densities of the material constituting the fluid (plasma) and the particle (erythrocytes) phases respectively; $(1-C) \rho_{f}$ is the fluid phase density and $C \rho_{p}$ is the particulate phase density, and the subscripts $f$ and $p$ denote the quantities associated with the plasma (fluid) and erythrocyte (particle) phases respectively. A constant particle volume fraction, $C$, is chosen since the particles are assumed to be in a small concentration (Batchelor, 1974, 1976; Srivastava, 1995). The suspension viscosity, $\mu_{s}$, has been chosen as the following empirical relation (Charm \& Kurland, 1974; Srivastava, 1983)

$$
\mu_{s}(C)=\frac{\mu_{0}}{1-m C},
$$


where

$$
m=7 \times 10^{-2} \exp \left[2.49 C+\left(\frac{1107}{T}\right) \exp (-1.69 C)\right] .
$$

Here $\mu_{0}$ is the fluid viscosity (suspending medium). Blood's temperature, $T$, is measured on the absolute scale $(K)$. Charm and Kurland (1974) performed experiments to investigate the suspension viscosity using a cone and plate viscometer. A good agreement was found within $10 \%$ of the tested cases (Charm \& Kurland, 1974). It was also found to be reasonably accurate for $C \leq 0.6$ (Charm \& Kurland, 1974; Srivastava \& Saxena, 1997; Srivastava, 1995). The expression for the drag coefficient of interaction, $S$, from Stokes drag for small-particle Reynolds number modified to account for the finite particulate fractional volume (Tam, 1969) is

$$
S=4.5\left(\frac{\mu_{0}}{a_{0}^{2}}\right) \frac{4+3\left(8 C-3 C^{2}\right)^{\frac{1}{2}}+3 C}{(2-3 C)^{2}},
$$

with $a_{0}$ as the red blood cell radius.

The flow is assumed to be steady with the additional conditions, $\delta / R_{o} \ll 1, \operatorname{Re}\left(2 \delta / L_{0}\right) \ll 1,2 R_{0} / L_{0} \sim \mathrm{O}(1)(\mathrm{L}$. Srivastava, 1983; V. Srivastava, 1983). Thus, the appropriate equations for flow in the core region are given as

$$
\begin{aligned}
(1-C) \frac{d p}{d z} & =(1-C) \frac{\mu_{s}(C)}{r} \frac{\partial}{\partial r}\left(r \frac{\partial}{\partial r}\right) u_{f}+C S\left(u_{p}-u_{f}\right), 0 \leq r \leq R_{1}, \\
C \frac{d p}{d z} & =C S\left(u_{f}-u_{p}\right), 0 \leq r \leq R_{1} .
\end{aligned}
$$

In the peripheral region, a Newtonian fluid is a suitable model for the plasma here. Assuming the flow is fully developed, steady, laminar, axisymmetric, and one-dimensional, the equation is written as (Young, 1968)

$$
\frac{d p}{d z}=\frac{\mu_{0}}{r} \frac{\partial}{\partial r}\left(r \frac{\partial}{\partial r}\right) u_{0}, \quad R_{1} \leq r \leq R .
$$

The boundary conditions (standard no slip conditions of velocities and the shear stresses at the tube wall and the interface) are given (Srivastava \& Saxena, 1997; Srivastava et al., 2010; Medhavi et al., 2012) as

$$
\begin{aligned}
u_{0} & =0, & \text { at } r & =R, \\
u_{0}=u_{f} \text { and } \tau_{p} & =\tau_{f}, & \text { at } r & =R_{1}, \\
\frac{\partial u_{f}}{\partial r}=\frac{\partial u_{p}}{\partial r} & =0, & \text { at } r & =0,
\end{aligned}
$$

where $\tau_{p}=\mu_{0} \partial u_{0} / \partial r$ and $\tau_{f}=(1-C) \mu_{s} \partial u_{f} / \partial r$ are the shear stresses of the peripheral and central regions respectively.

\section{Analysis}

The expressions for velocities, $u_{0}, u_{f}$ and $u_{p}$ are found to be

$$
\begin{aligned}
u_{0}= & -\frac{R_{0}^{2}}{4 \mu_{0}} \frac{d p}{d z}\left\{\left(R / R_{0}\right)^{2}-\left(r / R_{0}\right)^{2}\right\}, R_{1} \leq r \leq R \\
u_{f}= & -\frac{R_{0}^{2}}{4(1-C) \mu_{0}} \frac{d p}{d z}\left\{\mu^{\prime}\left[\left(R_{1} / R_{0}\right)^{2}-\left(r / R_{0}\right)^{2}\right]+(1-C)\left[\left(R / R_{0}\right)^{2}-\left(R_{1} / R_{0}\right)^{2}\right]\right\}, 0 \leq r \leq R_{1}, \\
u_{p}= & -\frac{R_{0}^{2}}{4(1-C) \mu_{0}} \frac{d p}{d z}\left\{\mu^{\prime}\left[\left(R_{1} / R_{0}\right)^{2}-\left(r / R_{0}\right)^{2}\right]+(1-C)\left[\left(R / R_{0}\right)^{2}-\left(R_{1} / R_{0}\right)^{2}\right]\right. \\
& \left.+\frac{4(1-C) \mu_{0}}{S R_{0}^{2}}\right\}, 0 \leq r \leq R_{1},
\end{aligned}
$$

where $\mu^{\prime}=\mu_{0} / \mu_{s}$.

The flow flux, $Q$, is now calculated as

$$
Q=2 \pi\left\{\int_{R_{1}}^{R} r u_{0} d r+\int_{0}^{R_{1}} r\left[(1-C) u_{f}+C u_{p}\right] d r\right\},
$$


which simplifies to

$$
Q=-\frac{\pi R_{0}^{4}}{8(1-C) \mu_{0}} \frac{d p}{d z}\left\{(1-C)\left[\left(R / R_{0}\right)^{4}-\left(R_{1} / R_{0}\right)^{4}\right]+\mu^{\prime}\left(R_{1} / R_{0}\right)^{4}+\beta\left(R_{1} / R_{0}\right)^{2}\right\}
$$

where $\beta=8 C(1-C) \mu_{0} / S R_{0}^{2}$, is a non-dimensional suspension parameter.

Since the sum of the fluxes in the two layers is equal to the total flux, one determines that $R_{1}=\alpha R$ and $\delta_{1}=\alpha \delta$ (Srivastava, 2007). Thus the pressure drop, $\Delta p(=p$ ar $z=0,-p$ at $z=L)$ across the stenosis is calculated from Equation (22) as

$$
\Delta p \quad=\int_{0}^{L}\left(-\frac{d p}{d z}\right) d z=\frac{8(1-C) \mu_{0} Q}{\pi R_{0}^{4}} \psi
$$

where

$$
\begin{gathered}
\psi=\int_{0}^{d}[\phi(z)]_{R / R_{0}=1} d z+\int_{d}^{d+\frac{L_{0}}{2}}[\phi(z)]_{R / R_{0}} \text { from Eq. (1) } d z+\int_{d+\frac{L_{0}}{2}}^{d+L_{0}}[\phi(z)]_{R / R_{0}} \text { from Eq. (2) } d z+\int_{d+L_{0}}^{L}[\phi(z)]_{R / R_{0}=1} d z, \\
\phi(z)=\frac{1}{\eta\left(R / R_{0}\right)^{4}+\alpha^{2} \beta\left(R / R_{0}\right)^{2}}, \\
\eta=(1-C)\left(1-\alpha^{4}\right)+\mu^{\prime} \alpha^{4} .
\end{gathered}
$$

In the expression for $\psi$, the third integral in closed form poses some difficulty so it will be evaluated numerically while the others are straight forward. Non-dimensionless expressions for the impedance (flow resistance), $\lambda$, the wall shear stress, $\tau_{w}$, the shear stress at the stenosis throat, $\tau_{s}$, are

$$
\begin{aligned}
& \lambda=(1-C)\left(\frac{1-L_{0} / L}{\eta+\alpha^{2} \beta}\right)+\frac{(1-C)}{L}\left(\frac{-R_{0} L_{0}}{2 \delta \alpha^{2} \beta}\right) \\
&\left\{1-\frac{1}{\left(1-\delta / R_{0}\right)}+\sqrt{\frac{\eta}{\alpha^{2} \beta}}\left[\tan ^{-1}\left(\sqrt{\frac{\eta}{\alpha^{2} \beta}}\right)-\tan ^{-1}\left(\sqrt{\frac{\eta}{\alpha^{2} \beta}}\left(1-\delta / R_{0}\right)\right)\right]\right\} \\
&+\frac{(1-C)}{L}\left(\frac{L_{0}}{2 \pi}\right) \int_{0}^{\pi} \frac{d \theta}{(a+b \cos \theta)^{2}\left[\eta(a+b \cos \theta)^{2}+\alpha^{2} \beta\right]} \\
& \tau_{w}=\frac{(1-C)}{\eta\left(R / R_{0}\right)^{3}+\alpha^{2} \beta\left(R / R_{0}\right)} \\
& \tau_{s}=\frac{(1-C)}{\eta\left(1-\delta / R_{0}\right)^{3}+\alpha^{2} \beta\left(1-\delta / R_{0}\right)}
\end{aligned}
$$

where

$$
\begin{aligned}
\lambda & =\bar{\lambda} / \lambda_{0}, \quad\left(\tau_{w}, \tau_{s}\right)=\left(\overline{\tau_{w}}, \overline{\tau_{s}}\right) / \tau_{0}, \\
\bar{\lambda} & =\Delta p / Q, \quad \overline{\tau_{w}}=-(R / 2)(d p / d z), \\
\overline{\tau_{s}} & =[-(R / 2)(d p / d z)]_{R / R_{0}=\left(1-\delta / R_{0}\right)}, \\
\lambda_{0} & =8 \mu_{0} L / \pi R_{0}^{4}, \quad \tau_{0}=4 \mu_{0} Q / \pi R_{0}^{3} .
\end{aligned}
$$

Here $\lambda_{0}$ and $\tau_{0}$ are the impedance and shear stress respectively for a Newtonian fluid in the absence of stenosis (i.e., $C=0$ ), and $\left(\bar{\lambda}, \overline{\tau_{w}}, \overline{\tau_{s}}\right)$ are (impedance, wall shear stress and shear stress at the stenosis throat) in their dimensional forms.

In the absence of the peripheral layer ( $\alpha=1)$ and particles in the blood ( $C=0$, Newtonian fluid), the following expressions are derived

$$
\begin{aligned}
\lambda_{N} & =1-\frac{L_{0}}{L}-\frac{R_{0} L_{0}}{6 \delta L}\left[1-\frac{1}{\left(1-\delta / R_{0}\right)^{3}}\right]+\frac{L_{0}}{2 \pi L} \int_{0}^{\pi} \frac{d \theta}{(a+b \cos \theta)^{4}}, \\
\tau_{w N} & =\frac{1}{\left(R / R_{0}\right)^{3}}, \\
\tau_{s N} & =\frac{1}{\left(1-\delta / R_{0}\right)^{3}} .
\end{aligned}
$$




\section{Numerical Results and Discussion}

Plots of the analytical solutions are provided for the following parameter values (Young, 1979), (MacDonald, 1979), (Shukla et al., 1980), (Joshi et al., 2009): $d=0 ; a_{0}=4 ; L_{0}=1 ; L=1,2,5 ; R_{0}=100,1000 \mu m$; $C=0,0.1,0.2,0.3,0.4,0.5,0.6 ; \delta / R_{0}=0,0.05,0.10,0.15,0.20 ; T=25.5^{\circ} C$, to observe the effects of the hematocrit, stenosis height, and the peripheral layer. The parameter $\alpha$ is computed using $\alpha=1-\varepsilon / R_{0}$ in which $\varepsilon \cong$ $\varepsilon(C)$ represents the peripheral layer of thickness as a function of cell concentration. In order for the computation to be done, the temperature was chosen as $T=25.5^{\circ} \mathrm{C}$ to be able to use Haynes' analysis (Guyton \& Hall, 2006) where $\varepsilon(\mu m)=6.18,4.67,3.60,3.12,2.58,2.18$ corresponds to hematocrit $(\%)=10,20,30,40,50,60$ respectively. The parameter $\mu^{\prime}$ is computed with the use of Equations (10) and (11). Simpson's rule is used to evaluate numerically the integral involved in Equation (24).

For any stenosis height $\left(\delta / R_{0}\right)$, the impedance $(\lambda)$ increases with the hematocrit $(C)$ (Figure 2 and Figure 3), which means that as the red blood cell count increases, the harder it is for the blood to flow due to the greater resistance to flow. Also, for any given hematocrit, as the stenosis height increases the impedance increases (Figure 2 and Figure 3). Thus as the stenosis increases in size radially, the greater the resistance to the flow which also increases the difficulty for the blood to flow in the blood vessels. In this two-layered model, as the tube length $(L)$ increases the impedance decreases (Figure 4), that is the longer the blood vessel remains without any branching occuring say, the easier it is for the blood to flow. Also, for any tube length, as hematocrit increases impedance increases (Figure 4) which again depicts that as the red blood cell count increases, the harder it is for the blood to flow with a composite stenosis being present.

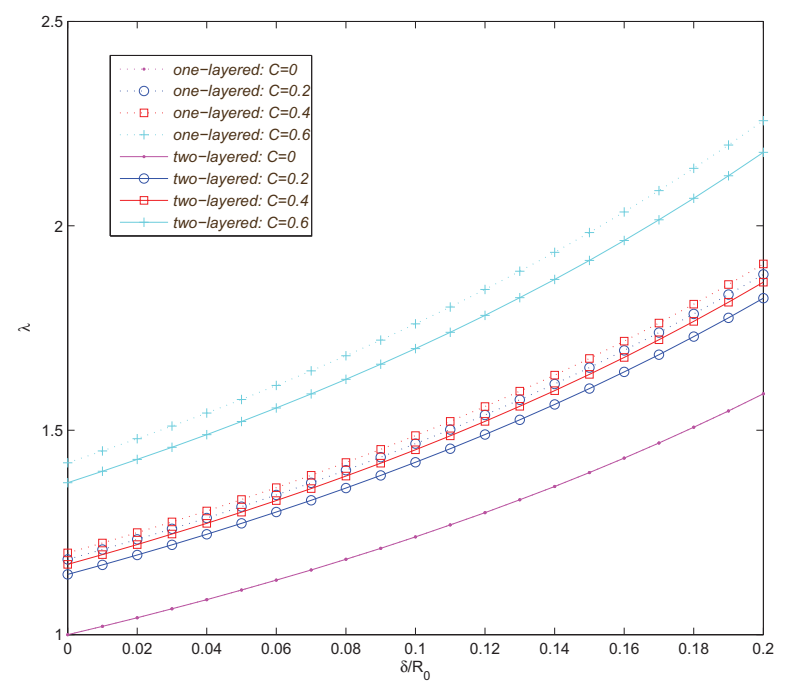

Figure 2. Impedance $\lambda$, versus stenosis height, $\delta / R_{0}$ for different hematocrits, $C$ (with $L=L_{0}=1, R_{0}=100 \mu m$ ) 


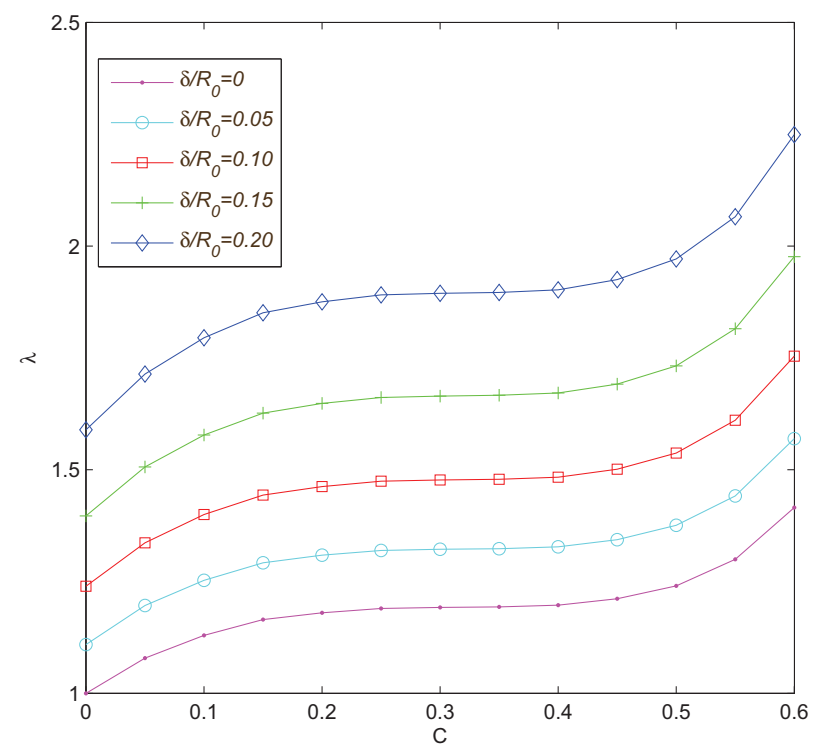

Figure 3. Impedance $\lambda$, versus hematocrit, $C$, for different stenosis heights, $\delta / R_{0}$ (with $L=L_{0}=1, R_{0}=1000 \mu m$ ) for this two-layered model

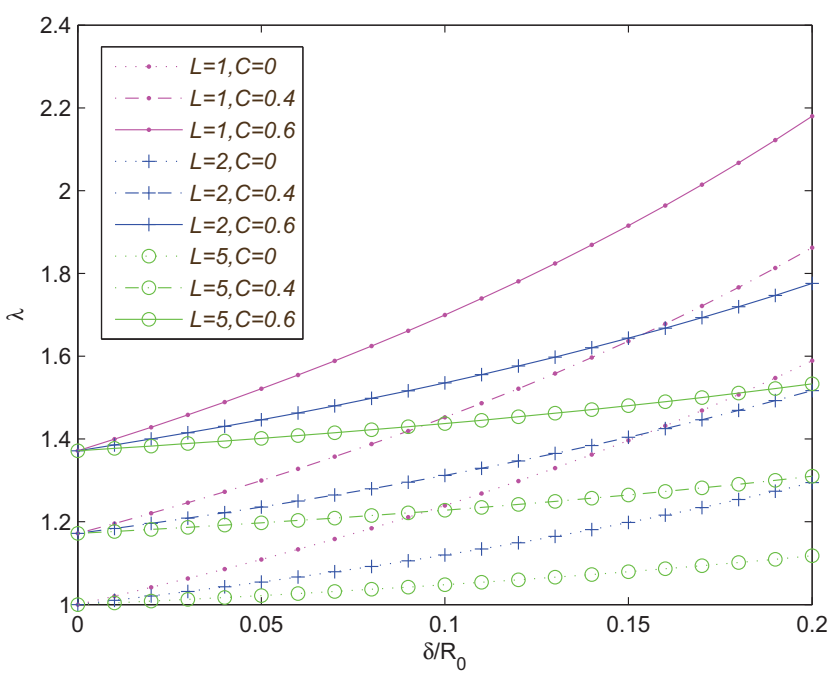

Figure 4. Impedance $\lambda$, versus stenosis height, $\delta / R_{0}$ for different artery lengths, $L$ (with $L_{0}=1, R_{0}=100 \mu m$ ) for this two-layered model

The shear stress on the wall $\left(\tau_{w}\right)$ increases rapidly in the upstream of the stenosis throat (located at $z=d+\frac{L_{0}}{2}$ ) and achieves its maximum at the throat of the stenosis before it begins to decrease downstream of the throat to the endpoint of the constriction profile (located at $\frac{z}{L_{0}}=1$ ), (Figure 5 and Figure 6). As the stenosis height increases the shear stress on the wall increases (Figure 5), which means that as the stenosis grows radially it causes greater damage to the walls of the vessels due to the increase of the shear stress that occurs. Furthermore, in the presence of a composite stenosis $\left(\delta / R_{0}=0.15\right)$, as the hematocrit increases the shear stress on the wall increases (Figure 6), thus the more red blood cells present in the vessel the greater the shear stress on the wall which in time can further damage the walls.

Trends in the shear stress at the stenosis throat $\left(\tau_{s}\right)$ and impedance $(\lambda)$ are similar with the variation of any of its parameters. For any stenosis height, as hematocrit $(C)$ increases the shear stress at the stenosis throat also increases (Figure 7 and Figure 8), which means that as the red blood cell count increases, the greater the shear stress at the 
stenosis throat. Also, shear stress at the stenosis throat increases with increasing stenosis height for any given hematocrit (Figure 7 and Figure 8). Thus as the stenosis increases in size radially, the greater the shear stress at the stenosis throat, which in the long term may lead to its rupture.

Additionally, in this analysis as the blood vessel diameter decreases, all three flow characteristics $\left(\lambda, \tau_{w}\right.$ and $\left.\tau_{s}\right)$ decrease (Figures 9-11). In general, the thickness of the walls of the blood vessels decreases from arteries to capillaries. Therefore as the blood vessels decrease in size, the walls can be thinner since these flow characteristics have a decreasing effect on them.

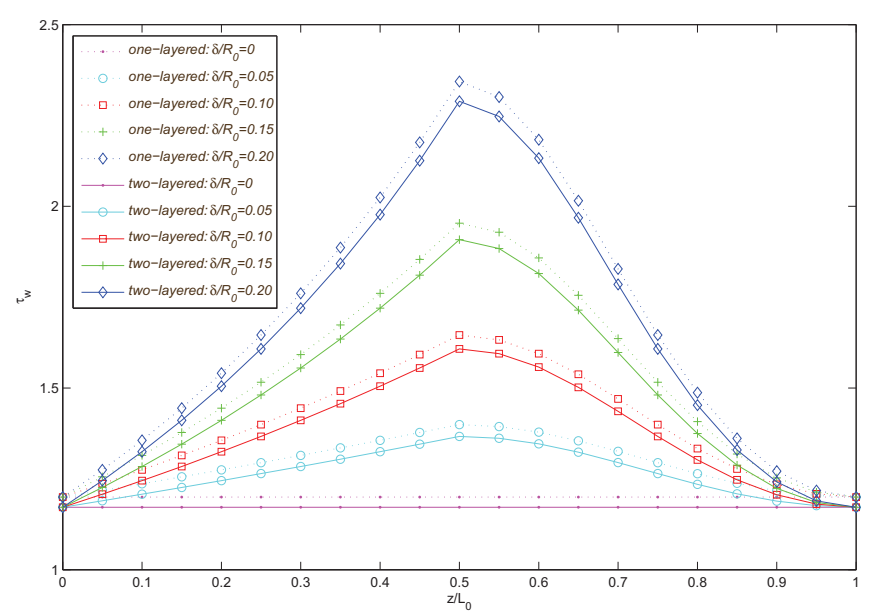

Figure 5. Wall shear stress distribution, $\tau_{w}$, in the stenotic region for different stenosis heights, $\delta / R_{0}$ (with $\left.C=0.4, R_{0}=100 \mu m\right)$

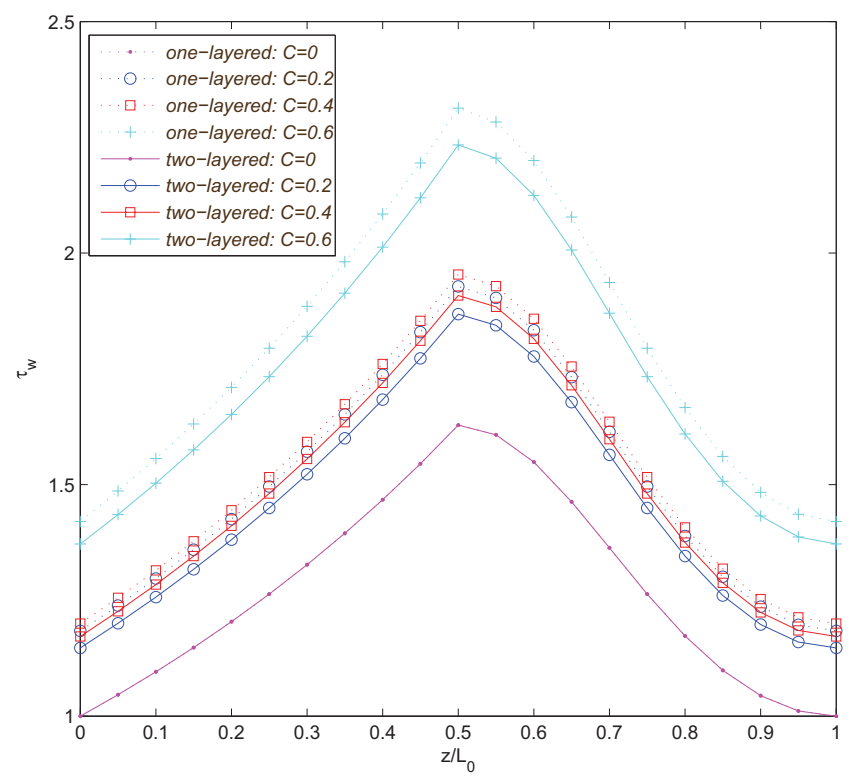

Figure 6. Wall shear stress distribution, $\tau_{w}$, in the stenotic region for different hematocrits, $C$ (with $\delta / R_{0}=0.15$,

$$
\left.R_{0}=100 \mu \mathrm{m}\right)
$$




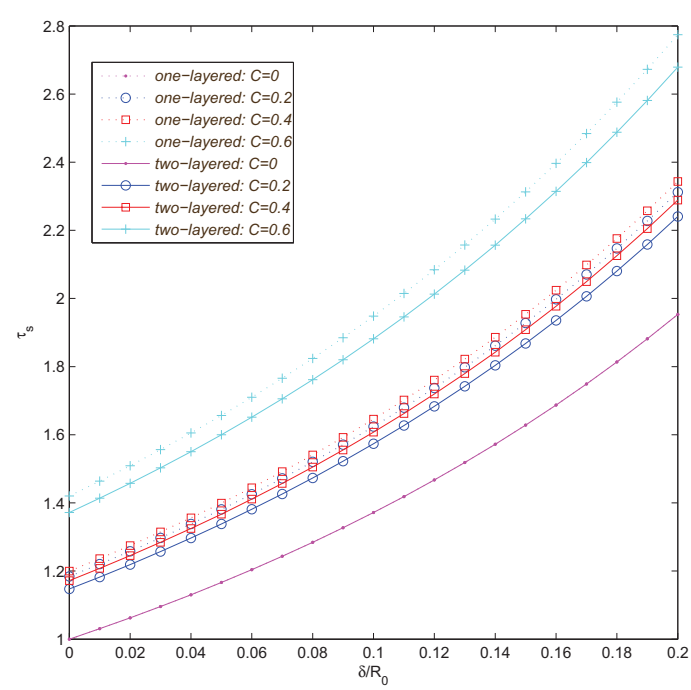

Figure 7. Shear stress at the stenosis throat, $\tau_{s}$, versus stenosis height, $\delta / R_{0}$ for different hematocrits, $C$ (with $\left.L=L_{0}=1, R_{0}=100 \mu m\right)$

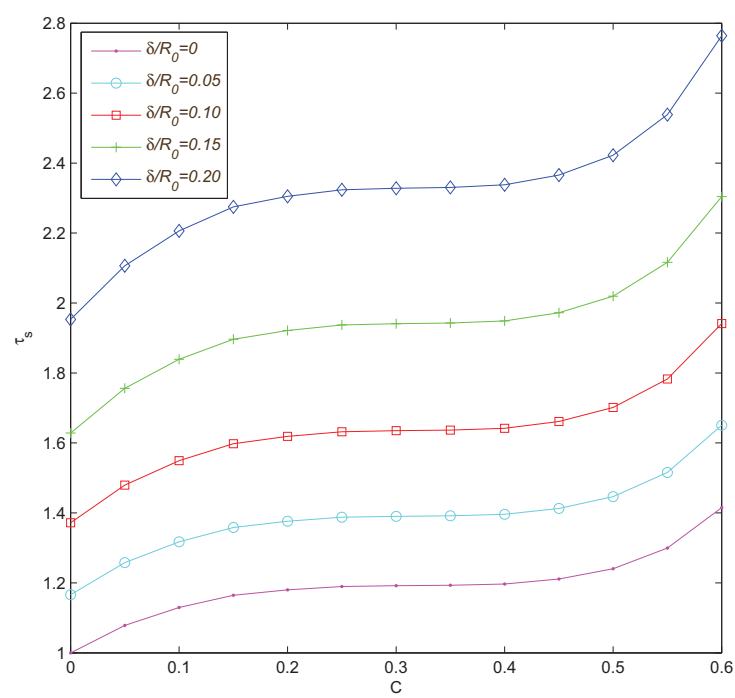

Figure 8. Shear stress at the stenosis throat, $\tau_{s}$, versus hematocrit, $C$, for different stenosis heights, $\delta / R_{0}$ (with $L=L_{0}=1, R_{0}=1000 \mu m$ ) for this two-layered model 


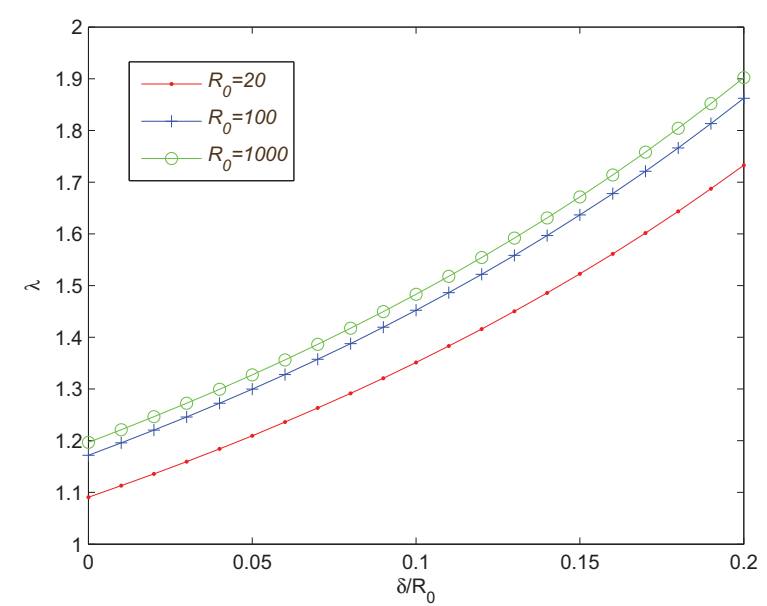

Figure 9. Impedance $\lambda$, versus stenosis height, $\delta / R_{0}$ for different radius of the tube $R_{0}$ (with $L=L_{0}=1, C=0.4$ ) for this two-layered model

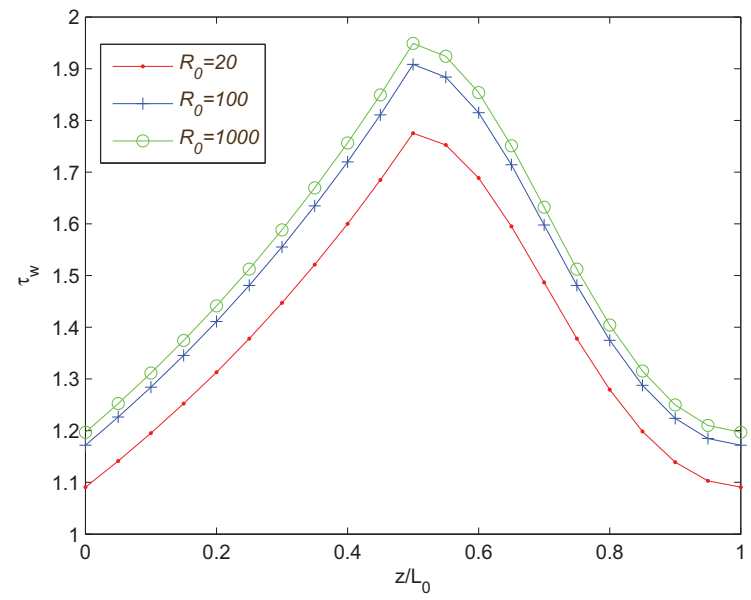

Figure 10. Wall shear stress distribution, $\tau_{w}$, in the stenotic region for different radius of the tube $R_{0}$ (with $\left.\delta / R_{0}=0.15, C=0.4\right)$ for this two-layered model

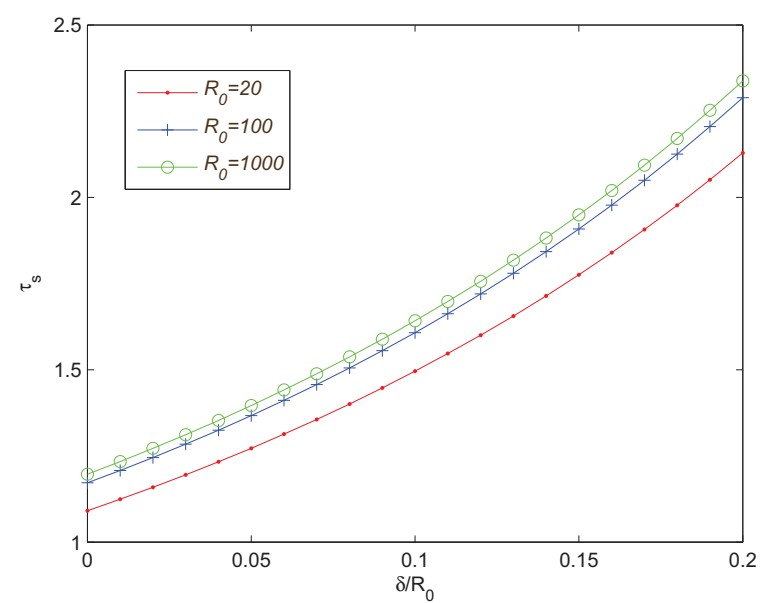

Figure 11. Shear stress at the stenosis throat, $\tau_{s}$, versus stenosis height, $\delta / R_{0}$ for different radius of the tube $R_{0}$ (with $L=L_{0}=1, C=0.4$ ) for this two-layered model 
In general, flow characteristics are found to be higher when an analysis of a particle-fluid suspension is conducted than when the fluid has no particles $(C=0)$ (Figures 2-4 and Figures 6-8). This is because in the particle-fluid suspension analysis, the red blood cells can no longer be ignored and their presence in the blood vessels will imply an increase in the impedance to flow and shear stress at both the vessel wall and stenosis throat.

The two-fluid model's flow characteristics are lower than those of the one-fluid model $(\alpha=1)$ (Figure 2 and Figures 5-7). That is in the two-layered model, these flow characteristics are significantly less than their corresponding values in the one-layered model for a two-phase macroscopic flow. Thus for small blood vessels with the presence of a layer of plasma present near to the walls, the effect of these flow characteristics decreases, thus decreasing the damage they may cause to the vessel in the presence of the composite stenosis. Note also that the thickness of the peripheral layer decreases as hematocrit increases since it depends on red cell concentration (Guyton \& Hall, 2006). Therefore the flow characteristics increases as hematocrit increases or as the peripheral layer's thickness decreases (Figure 3, Figure 6 and Figure 8), which can in time, damage in arterial vessels. This shows the increased need for the consideration of the peripheral layer (a two-layered flow) when examining blood flow through small blood vessels. The peripheral layer becomes particularly important in the study of diseased vessels since its thickness (together with the hematocrit value of the blood) varies for different diseases. Some of the popular diseases that occur are plasma cell dyscrasias (hematocrit $=28.00 \%, \alpha=0.816$ ), Hb SS-sickle cell (hematocrit $=24.80 \%$, $\alpha=0.795$ ), hypertension-controlled (hematocrit $=43.13 \%, \alpha=0.928$ ), hypertension-uncontrolled (hematocrit $=43.25 \%, \alpha=0.925)$ and polycythemia (hematocrit $=63.20 \%, \alpha=0.990)($ Bugliarello \& Sevilla, 1970).

\section{Conclusion}

In this analysis of blood flow in small vessels where a composite stenosis is manifested, a two layered model is used to model blood. The two layers are a peripheral plasma layer and a layer of erythrocytes suspended in plasma in the core region which is a suspension of all the erythrocytes in plasma (i.e., particle-fluid mixture). Three flow characteristics are examined. Flow impedance is found to increase with hematocrit and stenosis height but decreases with tube length. Therefore in the presence of a composite stenosis, the harder it is for the blood to flow if the number of red blood cells increases, the stenosis increases in size radially or a very short the blood vessel is being considered. The shear stress on the wall increases rapidly in the upstream of the stenosis throat, achieves its maximum at the throat of the stenosis then decrease downstream. It increases as stenosis height and hematocrit increases, that is if the number of red blood cells increases, the stenosis increases in size radially, the greater the stress on the wall which can in time damage the wall. Variations in the shear stress at the stenosis throat and impedance are similar with respect to all parameters. All three flow characteristics decrease when the blood vessel's diameter decreases.

In general, the flow characteristics were higher in the analysis of the particle-fluid suspension than in particle-free flow $(C=0)$ since the red blood cells can no longer be ignored and their presence in the blood vessels causes an increase in the impedance to flow and shear stress at both the vessel wall and stenosis throat. The two-fluid model's flow characteristics are lower than those of the one-fluid model. That is, the presence of a peripheral layer in this model reduces all three flow characteristics and any damage they may cause to the vessel with a composite stenosis. As the thickness of the wall decreases the flow characteristics increases which can in time can damage the arterial vessels. Therefore as a physiological application to the analysis, the peripheral layer becomes particularly important in the study of diseased vessels since the peripheral layer's thickness (and hematocrit in the blood) varies for different diseases. Some of the popular diseases which affect hematocrit and peripheral plasma layer thickness of the blood when they occur are plasma cell dyscrasias, Hb SS-sickle cell, hypertension and polycythemia.

Restrictions present (including the rigid wall, steady and fully developed flow, constant thickness of the peripheral layer) did not deter it from being an improvement over existing models with a composite stenosis found in the previous literature (Joshi et al., 2009; Medhavi et al., 2012). By considering a fully developed flow with $\delta / R_{0} \ll$ 1 , closed form solutions are obtained but this can only be applied to early vessel constriction (mild, composite stenosis). These solutions are used to find the relationships that the flow characteristics have with the variables which are indeed valid even though the parameter $\delta / R_{0}$ is limited to values up to 0.15 due to the possible flow separation that can occur even at small Reynold's numbers (Young, 1979).

Besides the above application of this study, the theory of particulate suspension can be used for analysis in areas such as powder technology, aerosol filtration, fluidization, environmental pollution, lunar ash flows, atmospheric fallout and combustion (Medhavi \& Singh, 2008). Particulate suspension theory has also been applied to improve the understanding of physiological flows such as protein diffusion, the movement of microorganisms and the particle deposition on the respiratory tract. The results obtained without considering the presence of a peripheral 
layer and stenosis can be used to analyse a mixture of particles in a fluid within a circular cylinder in any physical situation.

\section{References}

Batchelor, G. K. (1974). Transport properties of two-phase materials with random structure. Annual Review of Fluid Mechanics, 6, 227-255. http://dx.doi.org/10.1146/annurev.fl.06.010174.001303

Batchelor, G. K. (1976). Brownian diffusion of particles with hydrodynamic interaction. Journal of Fluid Mechanics, 74, 1-29. http://dx.doi.org/10.1017/S0022112076001663

Bitoun, J. P., \& Bellet, D. (1986). Blood flow through a stenosis in microcirculation. Biorheology, 23, 51-61.

Bugliarello, G., \& Sevilla, J. (1970). Velocity distribution and other characteristics of steady and pulsatile blood flow in fine glass tubes. Biorheology, 7, 85-107.

Chakravarty, S. (1987). Effects of stenosis on the flow behaviour of blood in an artery. International Journal of Engineering Science, 25, 1003-1010. http://dx.doi.org/10.1016/0020-7225(87)90093-0

Chaturani, P., \& Samy, R. P. (1986). Pulsatile flow of Casson fluid through stenosed arteries with applications to blood flow. Biorheology, 23, 499-511.

Charm, S. E., \& Kurland, G. S. (1974). Blood flow and Microcirculation. New York: John Wiley.

Cokelet, G. R. (1972). The Rheology of Human Blood: In Biomechanics. Prentice-Hall, New Jersey: Englewood Cliffs.

Drew, D. A. (1979). Stability of Stokes layer of a dusty gas. Physics of Fluids, 19, 2081-2081. http://dx.doi.org/10.1063/1.862517

Guyton, A. C., \& Hall, J. E. (2006). Textbook of Medical Physiology. Pennsylvania: Elsevier Saunders.

Haldar, K. (1985). Effects of the shape of stenosis on the resistance to flow through an artery. Bulletin of Mathematical Biology, 47, 545-550.

Haynes, R. H. (1960). Physical basis of the dependence of blood viscosity on tube radius. American Journal of Physiology, 198, 1193-1200.

Hersey, D., Byrnes, R. E., \& Roam, A. M. (1964). Blood rheology: temperature dependent of the power-law model. In Presented at the AICH, Meeting, Boston.

Joshi, P., Pathak, A., \& Joshi, B. K. (2009). Two-layered model of blood flow through composite stenosed artery. Applications and Applied Mathematics, 4, 343-354.

Lee, J. S., \& Fung, Y. C. (1970). Flow in locally-constricted tubes at low Reynolds number. Journal of Applied Mechanics Trans ASME, 37, 9-16. http://dx.doi.org/10.1115/1.3408496

MacDonald, D. A. (1979). On steady flow modelled cellular stenosis. Journal of Biomechanics, 12, 13-50. http://dx.doi.org/10.1016/0021-9290(79)90004-6

Mann, F. C., Herrick, J. F., Essex, H. E., \& Blades, E. J. (1938). Effects on blood flow of decreasing the lumen of blood vessels. Surgery, 4, 249-252.

Medhavi, A., \& Singh, U. K. (2008). A two-layered suspension flow induced by peristaltic waves. International Journal of Fluid Mechanics, 35, 258-272. http://dx.doi.org/10.1615/InterJFluidMechRes.v35.i3.40

Medhavi, A., Srivastava, R. K., Ahmad, Q. S., \& Srivastava, V. P. (2012). Two-phase arterial blood flow through a composite stenosis. e-Journal of Science and Technology, 7, 83-94.

Nerem, R. M. (1974). Fluid dynamics aspects of arterial disease. In Proceedings of a Specialist Meeting at Ohio University, 19-20.

Shukla, J. B., Parihar, R. S., \& Gupta, S. P. (1980). Effects of peripheral layer viscosity on blood flow thorugh an artery with mild stenosis. Bulletin of Mathematical Biology, 42, 797.

Shukla, J. B., Parihar, R. S., \& Rao, B. R. P. (1980). Effects of stenosis on non-Newtonian flow of blood in an artery. Bulletin of Mathematical Biology, 42, 283-294.

Skalak, R. (1972). Mechanics of microcirculation: In Biomechanics, Its Foundation and Objectives. New Jersey: 
Prentice-Hall, Englewood Cliffs.

Srivastava, L. M. (1985). Flow of a couple stress fluid through stenotic blood vessels. Journal of Biomechanics, 18, 479-485. http://dx.doi.org/10.1016/0021-9290(85)90662-1

Srivastava, L. M., \& Srivastava, V. P. (1983). On two-phase model of pulsatile blood flow with entrance effects. Biorheology, 20, 761-777.

Srivastava, V. P. (1995). Particle-fluid suspension model of blood flow through stenotic vessels. International Journal of Biomedical Computing, 38, 141-154. http://dx.doi.org/10.1016/0020-7101(94)01046-4

Srivastava, V. P. (2007). A theoretical model for blood flow in small vessels. Applications and Applied Mathematics, 2, 51-65.

Srivastava, V. P., Rastogi, R., \& Vishnoi, R. (2010). A two-layered suspension blood flow through an overlapping stenosis. Computers and Mathematics with Applications, 60, 432-441.

Srivastava, V. P., \& Saxena, M. (1994). Two-layered model of Casson fluid flow through stenotic blood vessels: applications to the cardiovascular system. Journal of Biomechanics, 27, 921-928. http://dx.doi.org/10.1016/0021-9290(94)90264-X

Srivastava, V. P., \& Saxena, M. (1997). Suspension model for blood flow through stenotic arteries with a cell-free plasma layer. Mathematical Biosciences, 139, 79-102. http://dx.doi.org/10.1016/S0025-5564(96)00130-7

Tam, C. K. W. (1969). The drag on a cloud of spherical particles in low Reynolds number flows. Journal of Fluid Mechanics, 38, 537-546. http://dx.doi.org/10.1017/S0022112069000322

Thurston, G. B. (1989). Plasma release cell-layering theory for blood flow. Biorheology, 26, 199-214.

World Health Organisation. (2013). The Atlas of Heart Disease and Stroke. Retrieved from http://www.who.int/cardiovascular_diseases/resources/atlas/en/

World Health Organisation. (2013). Cardiovascular diseases (CVDs). Retrieved from http://www.who.int/mediacentre/factsheets/fs317/en/index.html

Young, D. F. (1968). Effects of a time-dependent stenosis of flow through a tube. Journal of Engineering for Industry, 90, 248-254. http://dx.doi.org/10.1115/1.3604621

Young, D. F. (1979). Fluid mechanics of arterial stenosis. Journal of Biomechanical Engineering, 101, $157-175$. http://dx.doi.org/10.1115/1.3426241

\section{Copyrights}

Copyright for this article is retained by the author(s), with first publication rights granted to the journal.

This is an open-access article distributed under the terms and conditions of the Creative Commons Attribution license (http://creativecommons.org/licenses/by/3.0/). 